\title{
Knockdown Therapy for Treatment of Neoplasia in Draught Animals
}

\author{
Govind Mohan $^{1 *}$, P. Satheesh Kumar ${ }^{1}$, Sushil Kumar ${ }^{1}$, T.K. Bhattacharya ${ }^{2}$, \\ G. Anandasekaran ${ }^{3}$ and S. Karthiga ${ }^{3}$ \\ ${ }^{1}$ ICAR-National Dairy Research Institute, Karnal, Haryana-132001, India \\ ${ }^{2}$ ICAR-Directorate of Poultry Research, Rajendranagar, Hyderabad-500030 \\ ${ }^{3}$ ICAR-Indian Veterinary Research Institute, Izatnagar, Bareilly, Uttar Pradesh-243122 \\ *Corresponding author
}

\section{A B S T R A C T}

In India more than $50 \%$ of the total cultivated area is still being managed by using draught animals. With the modernization of agriculture, the use of tractors has increased but draught animals continue to be used on Indian farms. This mainly due to small land holdings and hill agriculture (G. Singh), India possessed the finest breeds of draught animals. Bullocks, buffaloes and camels are the major draught animals for field operations.

\section{Keywords}

Neoplasia, RNA Interference, knockdown therapy, Sema4D.

Article Info

Accepted: 30 June 2017 Available Online: 10 July 2017 Horses, mules, donkeys, yak and mithun are the pack animals for transport. Moreover, India has more number of draught breeds compare to very few milch breeds. The quality of work from the draught animals depends upon the power developed by them. In India draught animals were emotionally attached with the every farmer's life. Because Draught animals were helping farm works and with several stress condition giving constant Draught Power. Those adverse stress conditions leads to increase the incidence of neoplasia in draught animals which are higher compare to other utility animals in India. There are several factors for neoplasia and the range of neoplasia seen in draught animals is as diverse as that in human. Although more intensive therapeutic interventions, fatality rates remain unacceptably high, moreover not curing of cancerous in animals will be the chance of spread to humans through meat or milk or by direct contact. We are still lagging to solve those therapeutic interventions and fail to prevent the fatal due to neoplasia. Advance therapeutic prevention is therefore an important means of challenging this neoplasia. In this Review, we discuss the advances in the development of knockdown therapy and how we will efficiently utilise to cure the neoplasia in draught animals as well as other domestic animals and human from the Neoplasia.

\section{Introduction}

Information on draught ability on all the major Indian breeds of cattle, buffaloes and pack animals including cross breeds is scanty available, because major research programme has been undertaken on milch animals. Similarly the scenario of cancer incidences in India is not much clear due to lack of systemic study at national level and absence of Animal Cancer Registry. In India, the incidence of tumours is found to be highest in canines followed by equines and bovines. Singh et al., (1991) conducted a survey of tumours in domestic animals in Hisar and reported low occurrence of tumours in dogs (19.5\%) when compared to cattle $(53.56 \%)$. Udharwar et al., (2008) reported the twelve horn cancerous cattle and those animals surgical chemotherapy. National animal 
cancer seminar (2010) was conducted by Division of Pathology, IVRI, Izatnagar (UP) discussed about various advances in animal cancer research in India: diagnosis, treatment and clinical management. Very recently (2016) new siRNA based gene knockdown therapy developed and efficiently used in human cancer not in the veterinary cancer. This recent clinical studies that apply this technology to target gene knockdown in the neoplastic cells and promising approaches for developing siRNA constructs that knock down gene expression in target tissues beyond the neoplasia.

\section{Neoplasia and angiogenesis}

Neoplasia is the uncontrolled, abnormal growth of cells or tissues in the body and the abnormal growth itself called as neoplasm or tumour. It can be benign (non-cancerous form) or malignant (cancerous form). Benign types (skin mole, lipomas, and fibroids) are not life threatening unless malignant types or cancer (carcinoma, leukemia, lymphoma, sarcoma, and cancer of CNS) cause severe fatality in animals. Cancers still remains an enigmatic life threatening disease of human as well as animals, especially draught animals, despite the significant progress made in its diagnosis and treatment in recent years. This dreaded disease is a combination of different illnesses that hallmarks self-sufficiency in growth signal, insensitivity to antigrowth signal, evasion of apoptosis, limitless replicative potential, sustained angiogenesis and metastasis.

In body the vascular system and nervous system have several prominent anatomic similarities. The molecular mechanisms which are important for the specification, differentiation, and patterning of nerves also play an important role within the vasculature and vice versa (Carmeliet and TessierLavigne, 2005; Dickson, 2002; Eichmann et al., 2005). Development of the nervous system is regulated through the coordinated action of a variety of repulsive or attractive neuronal guidance factors, called "axonguiding molecules," that direct the growth of axons into specific pathways (Weinstein, 2005). Recently, these axon-guiding molecules (Semaphorins) have been shown to play a crucial role in the formation of vascular networks (Carmeliet and Tessier-Lavigne, 2005; Neufeld and Kessler, 2008).

Semaphorins have been found to function outside the nervous system serving as regulators of cell proliferation and migration and activators of lymphocytes (Kolodkin et al., 1993; Roney et al., 2013). Semaphorins and plexins are expressed in a variety of tissues besides the nervous system. Sema4D, a member of the class IV semaphorins, transduces a signal by directly binding to its high affinity receptor Plexin-B1. Emerging evidences has indicated that it also possesses a previously unrecognized function: a compensatory angiogenic factor which could promote tumor growth and angiogenesis (Ch'ng et al., 2007; Basile et al., 2004; Sakurai et al., 2010).

Sema4D is overe xpressed by some malignancies and plays a role in tumorinduced angiogenesis similar to vascular endothelial growth factor (VEGF), while the Sema4D-RhoAsignaling axis could recruit pericytes and regulate vascular permeability through endothelial production of PDGF-B and ANGPTL4, whereas VEGF lacks these effects (Zhou et al., 2014). Ding et al., (2016) investigated the influence of Sema4D on tumor growth and vascularity in colorectal carcinoma (CRC), especially in different VEGF backgrounds. They reported targeting Sema4D might serve as a parallel option for antiangiogenic therapy for colorectal cancer, particularly when traditional anti-VEGF therapies fail or tumours develop resistance to 
strategies targeting a single angiogenic signaling pathway. The results of this study suggest that Sema4D can play a significant role in tumor angiogenesis. RNA Interference of the Sema4D signaling pathway could be a valuable companion to anti-VEGF or other antiangiogenic therapies. Based on those studies we are proposes how we utilise that shRNA based Knockdown therapy efficiently for treatment of cancerous draught animals.

\section{RNA interference (RNAi)}

In the early 1990s, the first RNAi type phenomenon was reported in plant (van der Krol et al., 1990; Napoli et al., 1990). In 1992, Romano and Macino observed a similar phenomenon in Neurosporacrassa, which they referred to as "quelling" (Romano and Macino, 1992). A new hypothesis of RNA silencing was documented in animals first by Guo and Kemphues during their studies of antisense-mediated silencing in nematodes (Guo and Kemphues, 1995). In 2006, Andrew Fire and Craig C. Mello shared the Nobel Prize in Physiology or Medicine for their work on RNA interference in the nematode worm $C$. elegans. The 2123 bp small interfering RNA (siRNA) fragments generated by the processing of long dsRNA are reported to be the mediators of RNAi (Zamore et al., 2000). An endonuclease complex referred to as the RNA induced silencing complex (RISC) uses the siRNAs a guide to fragment the target mRNA of the homologous sequence resulting in a decrease in the mRNA levels. In mammals, dsRNAs longer than 30 basepairs are known to show non-specific suppression on overall mRNA translation (Stark et al., 1998). RNA interference (RNAi) is the process of sequence specific posttranscriptional gene silencing (PTGS) in animals and plants, the introduction of shorter dsRNA into mammalian cells induces the degradation of targeted mRNA with sequence specificity (Elbashiret al., 2001b).

\section{RNAi-mediated gene knockdown in cancer cells}

The siRNA-mediated Knockdown is performed in cultured cells using synthetic siRNAs or short hairpin RNA (shRNA)expressing vectors. Synthetic siRNA is often used to transiently knock down gene expression as its effects lasts typically lasting for 3 to 7 days (Elbashir et al., 2001a, b). The shRNA-expressing vectors are usually used to generate a stable inhibitory effect as they can integrate into the cell's genome (Paddison et al., 2002a; Martin and Caplen 2007). More recently, miRNA-expressing vectors have been also developed (reviewed in Scherr and Eder 2007).

siRNA, shRNA and miRNA-expressing vectors can be delivered to cells by electroporation, lipofection or microinjection. Viral vectors expressing shRNA can also be transduced into cells alternatively because of their genomic integration (Gupta et al., 2004). Analysis of knockdown efficiencies is mainly done by quantitative real-time PCR through measuring the remaining transcripts of the targeted genes. Protein levels can also be assessed by western blots.

\section{Materials and Methods}

\section{Cell culture}

Normal animal epithelial cell line or primary cell cull culture, (packaging cell 293T, human umbilical vein endothelial cells (HUVEC), and cancer cell lines, including HCT-116, Caco-2, LoVo, HT-29, COLO-205 also used). All cells were cultured in Dulbecco's Modified Eagle Medium (DMEM) (Thermo Fisher Scientific, Waltham, MA, USA) supplemented with $10 \%$ fetal bovine serum (Thermo Fisher Scientific), $2 \mathrm{mM}$ glutamine, $100 \mathrm{U}$ penicillin $/ 0.1 \mathrm{mg} / \mathrm{mL}$ streptomycin, and cultured in $5 \% \mathrm{CO} 2,37^{\circ} \mathrm{C}$ incubator. 


\section{Animal tissues}

Cancer samples were obtained from the affected animal. All samples were obtained from paraffin-embedded samples. Written consent was obtained from each participant patients. The study protocol should be approved by the institutional ethical committee.

\section{Design and production of siRNA}

Careful design of the siRNA is required to maximize silencing of the target gene (Sema4D) while minimizing off-target effects. Nevertheless, a number of siRNAs must be tested against different sites on a particular mRNA. In order to limit the off target effects of a particular siRNA, comparisons to annotated Sema4D mRNA databases should be made. The most common sources for Sema4D mRNA sequence information include the Reference Sequence (RefSeq) database, a comprehensive collection of non-redundant sequences (www.ncbi.nlm.nih.gov/ RefSeq/). A BLAST analysis is usually performed to ensure specificity of the designed siRNA (e.g., BLAST: www.ncbi.nlm.nih.gov/BLAST/) (Elbashir et al., 2002). Once the siRNA is designed, it can be produced by chemical synthesis, in vitro transcription and in vitro digestion of dsRNA using recombinant Dicer (Reynolds et al., 2006).

\section{Diagrammatic explanation of knockdown the Sema4D by RNAi}

a)

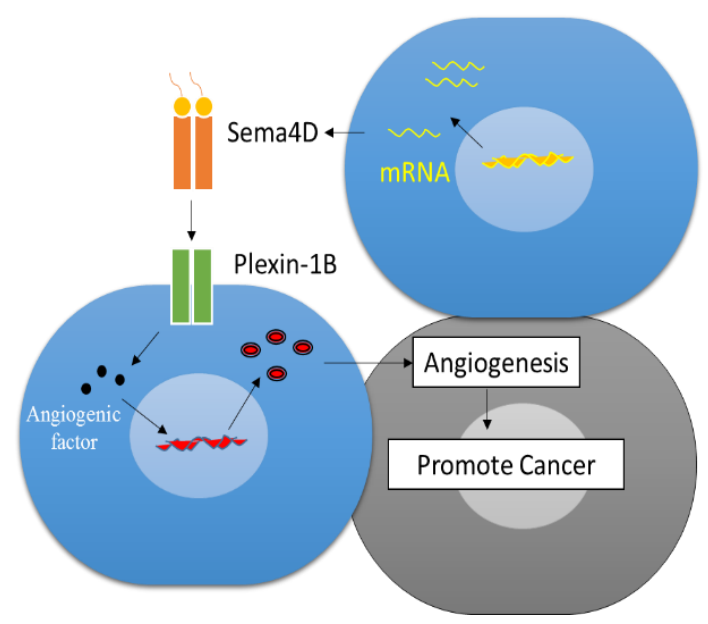

b)

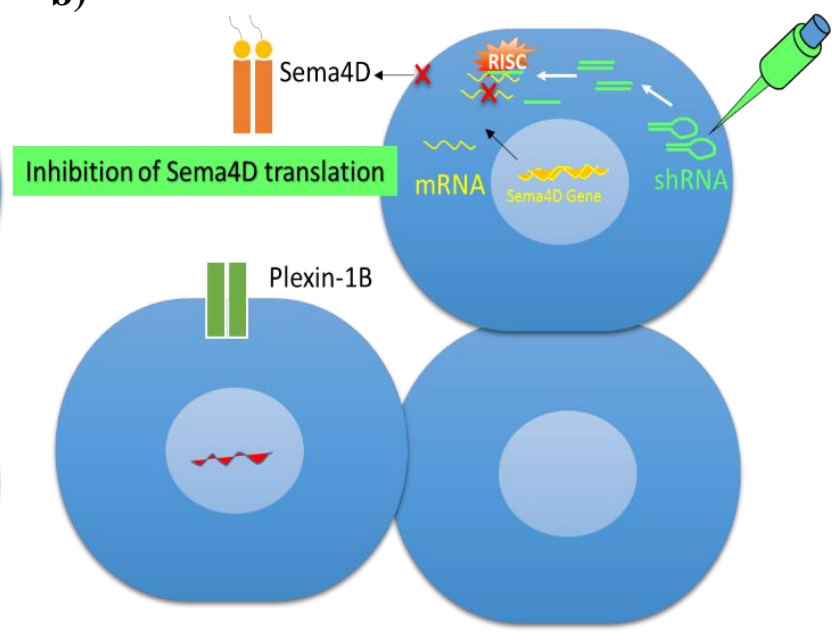

a) Sema4D-Plexin1B signaling pathway play a significant role in tumor angiogenesis which promote the cancer

b) Knockdown therapy- Exogenous anti-Sema4D shRNA targets to inhibiting the Sema4D mRNA translation which prevents the cancer formation

\section{Lentiviral vector system of gene transfer}

Gateway lentiviral vector system was first introduced by Professor John R Basile (Department of Oncology and Diagnostic Sciences, University of Maryland Dental
School, Baltimore, MD, USA) which was generous gift for us. The following lentiviral vectors were used by Ding et al., 2016: lentivirus coding for Sema4D short hairpin RNA (shRNA) (oligonucleotides based on the following sequence worked best to knock 
down Sema4D 5'GGCCTGAGGACCTTGCAGAAGA-3'), which was directed at knocking down the expression of Sema4D, and a Sema4D superexpressor coding lentivirus (Sema4D gene Accession: NM_006378.3, Forward primer: 5'-GCGGATCCATGAGGATGTGCACCCC3', reverse primer: 5'TGCGCGGCCGCTCAGTCTCCATCTGCG3 '), which helped to increase expression of Sema4D gene. Control lentiviruses were manufactured with enhanced green fluorescent protein expression vectors. Lentiviral vectors were packed and infections performed as previously reported (Naldini et al., 1996a; Naldini et al., 1996b).

\section{Results and Discussion}

Ding et al., (2016) reports that Immunohistochemical analysis of human CRC revealed high levels of Sema4D in a cell surface pattern, in all, $84.85 \%$ of CRC samples analyzed exhibited moderate to strong Sema4D expression. The positive ratios of Sema4D staining for well, moderately, and poorly differentiated cancers were $71.43 \%, 96.67 \%$, and $77.27 \%$, respectively. Sema4D is highly expressed in five different CRC cell lines, while VEGF expression level varies among these cell lines. HCT-116 showed the lowest VEGF level, while Caco-2 showed the maximum VEGF level. In vitro migration results show that regardless of cell type and VEGF background, Sema4D showed an enhanced in vitro proangiogenic effect to induce the migration of human umbilical vein endothelial cells. Finally, in vivo tumorangiogenic assays demonstrated that Sema4D alone can elicit a significant angiogenic response to promote tumor growth independently of VEGF.

Semaphorin-plexin interactions have already been implicated in a variety of responses, including epithelial cell contact modification and branching morphogenesis, regulation of pathological angiogenesis, tissue organization during development, and immune responses (Kolodkin et al., 1993; Roney et al., 2013). Over expression of Sema4D may provide tumors with the potential to support much denser cell populations, thus limiting the space for large fluid-filled cysts, and favoring the formation of much smaller fluid-filled cysts. Sema4D down regulation in these populations made the same cell lines unable to form tumors with dense cell populations. This may be explained by higher Sema4D levels enhancing angiogenesis through an increased blood supply, providing more resources for the dividing cells within that space. Tumors with lower Sema4D were less capable of driving neoangiogenesis in the tumor microenvironment, with an insufficient blood supply failing to support a large amount of cells in a limited amount of space.

In conclusion, the neoplasia may have more than one strategy to work against current antiangiogenic medicines, one of which could depend on the proangiogenic function of Sema4D. The results of this study suggest that Sema4D can play a significant role in tumor angiogenesis. Using shRNA knockdown therapy to interference of the Sema4D signaling pathway could be a valuable companion to anti-VEGF or other antiangiogenic therapies.

\section{Future prospects}

Cancer knockdown therapy is a rapidly emerging field of science promising targeted anticancer effect against several cancers. It has developed enough to find applications in human patients. But application in animals for treatment of cancer was not developed due to several reasons such as the cost of therapy, required expertise, investments, infrastructure, etc. Still, not yet being used in draught animals but hopefully it may applied widely in near future. Persistent scientific 
effort is need of the hour to bring these novel knockdown therapies in veterinary medicine. In the future, if we are to develop more effective and personalized knockdown therapies, we need to further decipher the complexities of tumor vascularization in animals. In addition, the application of this technology in veterinary medicine can also lead to development of novel animal models so that various gene knock down can be tested prior to use in animals.

\section{References}

Basile, J.R., Barac, A., Zhu, T., Guan, K.L., and Gutkind, J.S. 2004. Class IV semaphorins promote angiogenesis by stimulating Rho-initiated pathways through plexin-B. Cancer Res., 64(15): 5212-5224.

Basile, J.R., Castilho, R.M., Williams, V.P., Gutkind, J.S. 2006. Semaphorin 4D provides a link between axon guidance processes and tumor-induced angiogenesis. Proc. Natl. Acad. Sci. U S A, 103(24): 9017-9022.

Carmeliet, P., and Tessier-Lavigne, M. 2005. Common mechanisms of nerve and blood vessel wiring. Nature, 436: 193-200.

Ch'ng, E., Tomita, Y., Zhang, B., et al. 2007. Prognostic significance of CD100 expression in soft tissue sarcoma. Cancer, 110(1): 164-172.

Dickson, B.J. 2002. Molecular mechanisms of axon guidance. Sci., 298: $1959-1964$.

Ding, X., Qiu, L., Zhang, L., Xi, J., Li, D., Huang, X., Zhao, Y., Wang, X., and Sun, O. 2016. The role of semaphorin $4 \mathrm{D}$ as a potential biomarker for antiangiogenic therapy in colorectal cancer. OncoTargets and Therapy, 9: 1189-1204.

Eichmann, A., Le Noble, F., Autiero, M., and Carmeliet, P. 2005. Guidance of vascular and neural network formation. Curr. Opin. Neurobiol., 15: $108-115$.

Elbashir, S.M., Elbashir, S.M., Harborth, J., Lendeckel, W., Yalcin, A., Weber, K., and Tuschl, T. 2001a. Duplexes of 21 nucleotide RNAs mediate RNA interference in cultured mammalian cells. Nature, 41: 494-498.

Elbashir, S.M., Harborth, J., Weber, K., and Tuschl, T. 2002. Analysis of gene function in somatic mammalian cells using small interfering RNAs. Methods, 26: 199-213.

Elbashir, S.M., Lendeckel, W., and Tuschl, T. 2001b. RNA interference is mediated by 21- and 22-nucleotide RNAs. Genes Dev., 15: 188-200.

Guo, S., and Kemphues, K.J. 1995. par-1, a gene required for establishing polarity in C. elegans embryos, encodes a putative Ser/Thr kinase that is asymmetrically distributed. Cell, 81: 611-620.

Gupta, S., Schoer, R.A., Egan, J.E., Hannon, G.J. and Mittal, V. 2004. Inducible, reversible, and stable RNA interference in mammalian cells. Proc. Natl. Acad. Sci., 101: 1927-1932.

Kolodkin, A.L., Matthes, D.J., and Goodman, C.S. 1993. The semaphorin genes encode a family of transmembrane and secreted growth cone guidance molecules. Cell, 75(7): 1389-1399.

Martin, S.E., and Caplen, N.J. 2007. Applications of RNA interference in mammalian systems. Annu. Rev. Genomics Hum. Genet., 8: 81-108.

Naldini, L., Blomer, U., Gage, F.H., Trono, D., Verma, I.M. 1996. Efficient transfer, integration, and sustained long-term expression of the transgene in adult rat brains injected with a lentiviral vector. Proc. Natl. Acad. Sci. U S A, 93(21): 11382-11388.

Naldini, L., Blomer, U., Gallay, P., et al. 1996. In vivo gene delivery and stable transduction of nondividing cells by a lentiviral vector. Sci., 272(5259): 263267.

Napoli, C., Lemieux, C., and Jorgensen, R. 1990. Introduction of a Chimeric Chalcone Synthase Gene into Petunia Results in Reversible Co-Suppression of Homologous Genes in trans. Plant Cell, 2: 279-289.

National animal cancer seminar. 2010. 
Advances in animal cancer research in India: diagnosis, treatment and clinical management. Division of Pathology, IVRI, Izatnagar (UP).

Neufeld, G., and Kessler, O. 2008. The semaphorins: versatile regulators of tumour progression and tumour angiogenesis. Nat. Rev. Cancer, 8: 632645.

Paddison, P.J., Caudy, A.A., Bernstein, E., Hannon, G.J., and Conklin, D.S. 2002a. Short hairpin RNAs (shRNAs) induce sequence-specific silencing in mammalian cells. Genes Dev., 16: 948958.

Reynolds, A., Leake, D., Boese, Q., Scaringe, S., Marshall, W. S., and Khvorova, A. 2006. Rational siRNA design for RNA interference. Nat. Biotechnol., 22: 326330 .

Roney, K., Holl, E., and Ting, J. 2013. Immune plexins and semaphorins: old proteins, new immune functions. Protein Cell, 4(1): 17-26.

Sakurai, A., Gavard, J., Annas-Linhares, Y., et al. 2010. Semaphorin 3E initiates antiangiogenicsignaling through plexin D1 by regulating Arf6 and R-Ras. Mol. Cell Biol., 30(12): 3086-3098.

Scherr, M., and Eder, M. 2007. Gene silencing by small regulatory RNAs in mammalian cells. Cell Cycle, 6: 444-449.

Singh, G. 2000. Draught animal energy research in India. Proceedings of an ATNESA Workshop, September 1999, South Africa.
Singh, P., et al. 1991. Tumours in domestic animals in Hisar. Indian Vet. J., 68(8): 721-725.

Stark, G.R., Kerr, I.M., Williams, B.R., Silverman, R.H., and Schreiber, R.D. 1998. How cells respond to interferons. Annu. Rev. Biochem., 67: 227-64.

Udharwar, S.V., Aher, V.D., Yadav, G.U., Bhikane, A.U., and Dandge, B.P. 2008. Study on incidence, predisposing factors, symptomatology and treatment of horn cancer in bovine with special reference to surgery and chemotherapy. Vet. World, Vol. 1(1): 7-9.

Van der Krol, A.R., Mur, L.A., de, L.P., Mol, J.N., and Stuitje, A.R. 1990. Inhibition of flower pigmentation by antisense CHS genes: promoter and minimal sequence requirements for the antisense effect. Plant Mol. Biol., 14: 457-466.

Weinstein, B.M. 2005. Vessels and nerves: marching to the same tune. Cell, 120: 299-302.

Zamore, P.D., Tuschl, T., Sharp, P.A., Bartel, D.P. 2000. RNAi: double-stranded RNA directs the ATP-dependent cleavage of mRNA at 21 to 23 nucleotide intervals. Cell, 101(1): 25-33.

Zhou, H., Yang, Y.H., and Basile, J.R. 2014. The Semaphorin 4D-Plexin-B1-RhoA signaling axis recruits pericytes and regulates vascular permeability through endothelial production of PDGF-B and ANGPTL4. Angiogenesis, 17(1): 261274.

\section{How to cite this article:}

Govind Mohan, P. Satheesh Kumar, Sushil Kumar, T.K. Bhattacharya, G. Anandasekaran and Karthiga, S. 2017. Knockdown Therapy for Treatment of Neoplasia in Draught Animals. Int.J.Curr.Microbiol.App.Sci. 6(7): 4372-4378. doi: https://doi.org/10.20546/ijcmas.2017.607.455 